\title{
LA ACTUALIDAD DE ALGUNAS IDEAS DE UROS BACIC
}

Quienes hemos tenido el placer y el privilegio de ser alumnos del Doctor Uros Bacic recordamos su aprecio por el rigor científico y por la fundamentación metodológica. Tenemos presente su permanente preocupación por la correcta base gnoseológica de los estudios de la economía y su cuidado por el uso de los métodos sólidos de análisis. Las reflexiones que siguen procuran ser un reconocimiento a su labor de maestro y toman como tema algunas de sus ideas sobre cuestiones metodológicas que escuchamos en sus clases y que se reflejaron en sus trabajos. Muchas de esas ideas se encuentran en su artículo "Naturaleza de la Ley Económica" que se publicó en la revista Técnica y Economía (№ 12, juliodiciembre 1952), primera publicación de nuestra Universidad (en ese entonces Instituto Tecnológico del Sur). ${ }^{1}$ Es interesante notar que esta iniciativa de editar una publicación científica contó, entre otros, con la importante colaboración del Doctor Bacic, quizás un anticipo de su aporte posterior en la creación de nuestra revista Estudios Económicos.

Las reflexiones que siguen provienen de releer el trabajo mencionado y relacionar su contenido con la evolución de nuestra ciencia y particularmente de los estudios de Finanzas y Economía Pública. Y la conclusión general como veremos, será destacar ideas que Bacic defendía, su actualidad y la actitud científica que aprendimos a valorar y pretendemos seguir. El resto del trabajo se refiere a dos temas presentes en el mencionado artículo de Bacic: uno, aunque no tratado expresamente, es la concepción de nuestra disciplina como "Economía Política", el otro se relaciona con el método de la economía en comparación con el de las otras ciencias.

\section{EL CONCEPTO DE ECONOMIA POLITICA}

Una de las primeras cosas que se advierte en esta nueva lectura es la reiterada mención a la disciplina objeto de nuestro estudio como "Economía Polítican. Dicha insistencia no es una mera cuestión de gusto terminológico o de adhesión a una tradición denominativa. Responde a una concepción más profunda sobre el objeto de la materia. El tema no está específicamente tratado en este artículo pero resulta interesante verificar, por ejemplo, que en la primera página hay siete referencias a la ciencia económica y en cinco de ellas se la llama economía política. Esta constatación se une al recuerdo de la insistencia del Doctor Bacic en denominar Economía Política a las asignaturas del plan de estudios de nuestra carrera cada vez que tuvo participación en la discusión de dichos planes. 
Con la publicación del "Traité de l'economie politique" de Antoine de Monchréstien en 1615, nuestra disciplina adquirió una denominación que la acompañó durante más de dos siglos. Tal denominación se correspondía con el tratamiento de los temas, porque era habitual considerar los fenómenos económicos conjuntamente con las interrelaciones políticas con las que se asociaban. Se suele considerar a la "Investigación sobre la Naturaleza y Causa de la Riqueza de las Naciones" de Adam Smith como la primera obra "cientifica" de economía y es, sin duda, una obra de economía política plena de referencias a cuestiones institucionales y políticas, y a la influencia de tales cuestiones sobre los resultados sociales. A fines del siglo pasado, sin embargo, la economía como ciencia comienza a separarse de la política y, siguiendo un camino de especialización propio del desarrollo científico, circunscribe un objeto (el estudio de la escasez de acuerdo a la conocida definición de Lord Robbins) y elabora un cuerpo teórico de reconocida solidez que la ha convertido, para la opinión de muchos, en la más evolucionada de las ciencias sociales. Piénsese, por ejemplo, que es la única a la que anualmente se le otorga un Premio Nobel.

Una evolución paralela similar tuvieron los estudios de Hacienda Pública. Se trata obviamente de una disciplina que, por la propia naturaleza de su sujeto, es esencialmente "economía política". Así fue, sin duda, en la importante tradición italiana donde los nombres de Pantaleoni, De Viti de Marco, Einaudi, Grizziotti, Fasiani y, finalmente, Cosciani nos brindan una visión integrada de los fenómenos económicos y políticos. Los libros con que estudiábamos Finanzas Públicas en los años 60 contenían abundantes referencias a los procesos de decisión política y cómo los instrumentos financieros debían ser considerados en relación a los mismos. Algunos de ellos, incluso, contenían modelos alternativos de comportamiento estatal que se derivaban de las ideas originales de De Viti de Marco. ${ }^{2}$ Pero la corriente predominante se apartó de esta tradición y siguiendo las líneas trazadas, principalmente, por Pigou y Dalton relegaron las cuestiones políticas propias de la economía pública a la romántica noción de un gobierno benevolente y omnisciente sólo interesado en maximizar una función de bienestar social. ${ }^{3} \mathrm{La}$ "despolitización" de la economía llegó entonces, paradójicamente, al campo de la economía pública.

Por el mismo tiempo en que la economía como ciencia se desprendía de la política tanto en la denominación de la disciplina como en sus contenidos, la economía real comenzaba a politizarse en forma creciente. También a fines del siglo pasado el hacendista alemán Adolfo Wagner enunció una "ley de la creciente extensión de la actividad estatal", señalando que "... las necesidades estatales se hallan en crecimiento regular en los países que se desarrollan .... ${ }^{4}$ Los hechos confirmaron la predicción implícita en la ley de Wagner, aunque quizás no por las razones que él sostenía. Pero lo cierto es que el indicador más habitual, la relación entre el gasto público y el producto nacional, creció en los principales países desarrollados de menos del $10 \%$ en los tiempos de los escritos de Wagner a más del $50 \%$ cien años después.

El desarrollo de la economía "pura", como señalamos antes, permitió la progresiva construcción de un importante aparato analítico que fue arrojando luz 
sobre los comportamientos en el marco de los intercambios del mercado. Pero, en cambio, no contribuyó a la comprensión de los fenómenos político-económicos, que cada vez eran más importantes a medida que las decisiones económicas se politizaban. El problema fundamental estaba en el abandono de la política en las explicaciones de las decisiones públicas, en el uso de un modelo exógeno e ingenuo de comportamiento estatal. Ya por los años 70, la crisis de las recomendaciones politicas de la macroeconomía keynesiana y del estado de bienestar, pusieron en cuestión la capacidad del aparato analítico de la economía convencional para comprender el funcionamiento de un sistema económico con tan amplia participación del sector público.

Por entonces fué ganando aceptación lo que en la terminología de Kuhn podriamos denominar un cambio de paradigma. La insuficiencia del enfoque teórico convencional para explicar la realidad de una economía altamente politizada dio lugar al surgimiento de una nueva visión que reincorporaba los elementos de la política al estudio de las cuestiones de la economía pública. La idea fundamental es endogeneizar el comportamiento de los entes colectivos. Se trata de una vuelta a la economia política.

Como es sabido, este cambio de paradigma está principalmente vinculado a la obra de James Buchanan, aunque reconoce dos importantes antecedentes que es necesario mencionar: las ideas de Wicksell y de Schumpeter. ${ }^{5}$ Wicksell proporcionó una primer idea básica. Los economistas deben dejar de lado la perspectiva de que su tarea es dar consejos acerca de cómo llegar a los objetivos determinados exteriormente por un déspota benevolente. Para eso deben endogeneizar el comportamiento politico, considerar que la politica es una actividad humana análoga a la económica, llevada a cabo por las mismas personas, que actuan, en ambos campos, con sus intereses y sus valores. ${ }^{6}$ Por su parte, Schumpeter ${ }^{7}$ aportó la idea, posteriormente desarrollada extensamente por Downs ${ }^{\delta}$, según la cual la esencia de la democracia reside en la competencia electoral para acceder al poder.

La concepción fundamental de esta "Nueva Economía Política" consiste en considerar tanto a la economía como a la politica como dos formas básicas de intercambio. Las personas procuran obtener cosas que normalmente no pueden lograr por sí mismas. Recurren, entonces, a la división del trabajo y a intercambios. Estos pueden ser acordados por contratos voluntarios (economía) o coactivos (política). La teoría económica pura desarrolló, como hemos dicho, un importante aparato analítico para estudiar los intercambios voluntarios del mercado. Las mismas bases metodológicas pueden ser utilizadas para estudiar los intercambios colectivos a través de procesos de naturaleza política. Podemos decir que nuevamente tenemos una paradoja. Cuando vuelven a relacionarse economía y política lo hacen con el método que la economía desarrolló en su periodo de apartamiento.

Las Finanzas Públicas, o la Economía Pública según la denominación que parece extenderse, en nuestros dias contempla una fructífera renovación con la creciente incorporación del nuevo enfoque como puede apreciarse por el número de trabajos publicados en las revista científicas ${ }^{10}$, el creciente espacio que le otorgan los Manuales destinados al dictado de los cursos universitarios, los nuevos programas de investigación que se proponen y la perspectiva de unificación metodológica de 
las ciencias sociales que el nuevo enfoque sugiere. ${ }^{11}$

\section{LA ECONOMIA COMPARADA CON OTRAS CIENCIAS}

El propósito general del trabajo del Dr. Bacic, que inspiró estas notas, consiste en encontrar respuestas a una tradicional cuestión. ¿La economía, y en general las ciencias que se ocupan del comportamiento humano, pueden ser consideradas en un mismo plano con las que se ocupan de los hechos naturales? ¿Son esencialmente análogas sus bases metodológicas y la naturaleza de sus hallazgos o existen diferencias fundamentales? ¿Qué alcance pueden tener los elementos comunes de disciplinas distintas, especialmente de las ciencias que se ocupan de los actos humanos (libres, conscientes, individualmente impredecibles) comparadas con ciencias que estudian hechos que están más allá de la acción humana?

Bacic analiza la cuestión a través de una reflexión sobre la naturaleza de la ley económica. Se pregunta hasta qué punto se puede asimilar la ley económica a la ley natural, es decir, hasta qué punto la economía política es una ciencia como las de la naturaleza, y para responder examina los principales puntos del desarrollo histórico del concepto. ${ }^{12}$

Para Bacic una de las causas de las divergencias ha residido en dar a la ley económica un concepto valorativo, error que señala en clásicos y fisiócratas. En términos actuales diríamos confundir lo positivo con lo normativo, los hechos con los valores. Pero aún en lo estrictamente referido al estudio de los hechos, muchas veces se ha errado al dar el carácter de ley a proposiciones no suficientemente fundadas Las dificultades para formular las leyes económicas que ayuden a la comprensión de los fenómenos se refieren a la complejidad del fenómeno, al entrelazamiento con otros hechos sociales, a la imposibilidad de experimentación. Pero todo esto no justifica la tesis de la separación metodológica básica entre ciencias humanas y naturales.

Bacic distingue entre necesidad empírica y lógica de la ley, y demuestra el carácter científico de la economía política, reconociendo las dificultades que surgen de la libertad humana y de la imprevisibilidad de las conductas, reduciendo la necesidad empírica a regularidades estadísticas y afirmando la necesidad lógica y el uso del supuesto de conducta racional.

Sus conclusiones se basan, en nuestra opinión, en distinguir la validez lógica de las proposiciones y su corroboración empírica:

"El análisis cumplido nos lleva a la conclusión de que las generalizaciones elaboradas en el dominio de la ciencia económica ... pueden ser acercadas a las que se formulan en las disciplinas científico-naturales. Ambas categorias de claboraciones científicas expresan, en efecto, relaciones necesarias" ${ }^{13}$

Es decir, para Bacic no hay diferencias sustanciales respecto a la elaboración de los modelos teóricos con los que pretendemos comprender tanto el comportamiento humano en economía como los fenómenos naturales. Pero sí hay diferencias cuando contrastamos los modelos teóricos con la realidad:

" ¿Nos autoriza la presencia de tales rasgos comunes a cumplir un paso ulterior e identificar a la ley económica con la ley natural? La contestación no puede ser 
sino negativa. La divergencia radical entre la naturaleza de los términos que vinculan las leyes económicas y la de los que enlazan las leyes naturales ... (aconseja) ... no perder de vista las divergencias de orden cuantitativo que existen entre ambas categorías de leyes, desde el punto de vista de su aplicabilidad. La ley económica presenta, en efecto, una validez gnoseológica inferior a la de la ley natural y no constituye, por tanto, sino un instrumento menos eficaz a la afirmación del hombre sobre la realidad. Pese a todos los esfuerzos que los economistas han cumplido ... la distancia que separa la ley económica de la realidad es todavía notablemente mayor de la que existe entre la ley natural y el mundo de la naturalezan ${ }^{14}$

Pero estas diferencias, esta aproximación más dificultosa a una realidad más compleja «... no autoriza inferir, comparándo la ley económica a la natural, una dignidad inferior a la primera". ${ }^{15}$

Se deben reconocer las limitaciones de aplicabilidad de las generalizaciones económicas al mundo real, pero esas limitaciones también las tienen las ciencias naturales. Las teorias necesariamente son abstracciones y tal reconocimiento tampoco permite diferenciar a la ley económica como una cosa distinta de la ley natural:

"... tal diferencia no es sino gradual y tampoco ofrece un criterio de discriminación riguroso entre estos dos grupos de generalizaciones científicas" ${ }^{16}$

\section{LA ACTUALIDAD DE LAS IDEAS DE BACIC}

Podemos sostener que la corriente principal de nuestra ciencia comparte las ideas básicas que nuestro autor exponía en 1952.

En primer lugar, lo que se ha denominado monismo metodológico prevalece en el pensamiento científico de hoy. ${ }^{17}$ No existe una discontinuidad metodológica fundamental entre ciencias humanas y naturales aunque haya diferencias de grado en la aplicación.

Una segunda idea básica se relaciona con la forma en que encaramos el conocimiento científico, que es análoga en todas las ciencias. Las ciencias se componen de teorias, que son construcciones lógicas elaboradas por el pensamiento humano, con las cuales pretendemos formular las generalizaciones que nos permitan comprender los fenómenos y con las cuales pretendemos contrastar el mundo real. En palabras de Franz Kreuzer "... el mundo nos entra por la vista sólo en apariencia; de hecho nos sale por la miradan. ${ }^{18}$ Las generalizaciones o leyes de la economía se basan en hipótesis teóricas, pero, dice Bacic:

"... también la ley natural descansa en un supuesto general, en una hipótesis". ${ }^{19}$

El tercer aspecto importante se refiere a la característica general de las hipótesis teóricas en economía: su base en el comportamiento de las personas (individualismo metodológico) que procuran sus objetivos ponderando las alternativas a su alcance (racionalidad instrumental).

En efecto, para Bacic "... tampoco ha sido elegido con arbitrariedad el supuesto fundamental en que, descansa la economia deductiva. Si los sujetos 
económicos no se comportan siempre de modo perfectamente racional, el principio económico continúa, no obstante, dominando ampliamente las unidades de consumo y sobre todo las de producción". ${ }^{20}$

Incluso cuando Bacic habla de lo que podriamos interpretar como excepciones al comportamiento racional apela a su defensa como regularidad estadística cuando dice:

"... los bienes son escasos ... los hombres tienden a procurárselos generalmente con el menor sacrificio ... todos testimonian de una mayor o menor conducta racionaln. ${ }^{21}$

Estos párrafos parecen un alegato de los autores actuales que defienden la extensión del paradigma económico a otras ciencias sociales, según lo hemos señalado precendentemente.

El artículo que comentamos fue escrito hace cuarenta años y su lectura de hoy permite afirmar, en nuestra opinión, su actualidad. En efecto, la evolución metodológica de la ciencia económica en esos años, sus logros, su ubicación dentro del panorama general de las ciencias, el renacimiento del concepto de economía política y la extensión de las herramientas del análisis económico a otros campos del comportamiento humano prueban el acierto de sus ideas.

\author{
Ricardo Enrique Bara \\ Departamento de Economía \\ Universidad Nacional del Sur
}

\title{
NOTAS
}

1) Podemos decir que el trabajo del Doctor Bacic "Naturaleza de la Ley Económica". Témnica y Economía. № 12. Instituto Tecnológico del Sur, Bahía Blanca, 1952, páginas 125-135, es uno de los primeros trabajos científicos sobre economia originado en nuestra Universidad.

2) Véase, al respecto, Antonio De Viti de Marco (1934). Principios Fundamentales de Economía Financiera. Editorial Revista de Derecho Privado. Madrid, y Mauro Fasiani (1962), Principios de Ciencia de la Hacienda. Editorial Aguilar, Madrid.

3) James Buchanan (1979). Politics without Romance: A Sketch of Positive Public Choice Theory and its Normative Implications, IHS Journal. vol. 3, Physica-Verlag, Viena.

4) Wagner, Adolfo (1877), Finanzwissenschaft, Leipzig. citado por Richard Bird (1974), La Dinámica del Gasto Público, Instituto de Estudios Fiscales, Madrid, p. 27 y 28.

5) Véase Knut Wicksell (1896), A New Principle of Just Taxation, en Musgrave R. y Peacock A. (eds.) (1964), Classics in the Theory of Public Finance, Macmillan \& Co, Londres, y Schumpeter, Joseph (1963), Capitalismo, Socialismo y Democracia, editorial Aguilar. Madrid. capitulo 21 y 22.

6) Véase James Buchanan (1986). La Constitución de la Politica Económica, discurso pronunciado en ocasión de recibir el Premio Nobel de Economia, publicado en Papeles de Economía Española (1987). volumen 30-31, Madrid.

7) Schumpeter (1963), op. cit., p.343.

8) Véase Anthony Downs (1973), Teoria Económica de la Democracia, editorial Aguilar, Madrid.

9) Esta es la denominanción que, por ejemplo. Robert Inman asigna al nuevo enfoque. Ver A. Auerbach y M. Feldstein, eds. (1987), Handbook of Public Economics, volumen Il, capitulo 12, North-Holland. Amsterdam. 10) Véase, por ejemplo, el detalle publicado con motivo de los 25 años de la aparición de Public Choice, la primera revista que se especializó en los temas de la intersección de la economía y la política (Public Choice, volumen $71, n^{\circ} 3$, september 1991).

11) $\mathrm{Al}$ respecto es interesante señalar estas observaciones de Buchanan sobre una convergencia interdisciplinaria: “El científico político tanto como el economista están crecientemente atraídos por el 
nuevo campo, Así, los teóricos de los juegos, los psicólogos de la organización, los sociológos de los grupos pequeños, los antropólogos interesados en los grupos cooperativos, forman un conjunto del que está surgiendo un sistema de comunicación interdisciplinario. Esta interdependencia especializada si, en los hechos, llega a ser general a varias especializaciones emergentes, puede esperarse que de lugar, finalmente, a movimientos hacia una ruptura de las fronteras tradicionales de las disciplinas y al desarrollo de nuevos paradigmas revolucionarios”. Citado por David Reisman (1990), The Political Economy of James Buchanan, Texas A\&M University Press.

En el mismo sentido puede interpretarse la obra de Gary Becker y la reciente publicación de Bruno Frey (1992), Economics as a Science of Human Behavior: Towards a New Social Science Paradigm, Kluwer Academic Publisher, Boston, que procura demostrar no sólo la aplicabilidad de la economía a los problemas de investigación en ciencia política, sociología, psicología y derecho, sino también por qué puede servir de paradigma para unificar el estudio del comportamiento humano.

12) Bacic (1952): op. cit. página 126.

13) Bacic (1952): op. cit. página 135.

14) Bacic (1952): op. cit. página 135.

15) Bacic (1952) op. cit. página 132.

16) Bacic (1952): op. cit. página 135.

17) Para un desarrollo más ampljo del tema puede consultarse Juan José Rubjo Guerrero (1990): La lógica de la investigación científica en Ciencia Sociales, en Economía y Hacienda Pública, en Hacienda Pública Española, № 115 , páginas 13-42.

18) El Porvenir está Abierto (Conversaciones entre Konrad Lorenz y Karl Popper), Tusquets, Barcelona, 1992. página 39.

19) Bacic (1952): op. cit. página 132.

20) Bacic (1952): op. cit. página 132.

21) Bacic (1952): op. cit. página 130.

\section{REFERENCIAS BIBLIOGRAFICAS.}

BACIC, UROS (1952): "Naturaleza de la Ley Económican, Técnica y Economía, № 12. Instituto Tecnológico del Sur, Bahia Blanca, páginas 125-135.

BIRD. RICHARD (1974): La Dinámica del Gasto Público, Instituto de Estudios Fiscales, Madrid.

BUCHANAN, JAMES (1979). Politics without Romance: A Sketch of Positive Public Choice Theory and its Normative Implications, IHS Journal, vol. 3, Physica-Verlag, Viena.

DE VITI DE MARCO. ANTONIO (1934). Principios Fundamentales de Economía Financiera, Editorial Revista de Derecho Privado, Madrid.

FASIANI, MAURO (1962). Principios de Ciencia de la Hacienda, Editorial Aguitar. Madrid.

KREUZER, FRANZ (1992): El porvenir está abierto. Tusquets, Barcelona.

REISMAN, DAVID (1990). The Political Economy of James Buchanan, Texas A\&M University Press.

RUBIO GUERRERO, J.J. (1990): La lógica de la investigación científica en Ciencia Sociales, en Economia y Hacienda Pública, en Hacienda Pública Española, № 115, páginas 13-42.

SCHUMPETER, JOSEPH (1963), Capitalismo, Socialismo y Democracia, editorial Aguilar. Madrid.

WICKSELL. KNUT (1896), A New Principle of Just Taxation, en Musgrave R. y Peacock A. (eds.) (1964), Classics in the Theory of Public Finance, Macmillan \& Co. Londres. 